\title{
RADIUS OF STARLIKENESS FOR CLASSES OF ANALYTIC FUNCTIONS
}

\author{
KANIKA KHATTER, SEE KEONG LEE, AND V. RAVICHANDRAN
}

\begin{abstract}
We consider normalized analytic function $f$ on the open unit disk for which either $\operatorname{Re} f(z) / g(z)>0,|f(z) / g(z)-1|<1$ or $\operatorname{Re}\left(1-z^{2}\right) f(z) / z>0$ for some analytic function $g$ with $\operatorname{Re}\left(1-z^{2}\right) g(z) / z>0$. We have obtained the radii for these functions to belong to various subclasses of starlike functions. The subclasses considered include the classes of starlike functions of order $\alpha$, lemniscate starlike functions and parabolic starlike functions.
\end{abstract}

\section{INTRODUCTION}

For any two classes $\mathcal{G}$ and $\mathcal{H}$ of analytic functions defined on the unit disk $\mathbb{D}$, the $\mathcal{H}$-radius for the class $\mathcal{G}$, denoted by $\mathcal{R}_{\mathcal{H}}(\mathcal{G})$, is the maximal radius $\rho \leq 1$ such that the $f \in \mathcal{G}$ implies that the function $f_{r}$, defined by $f_{r}(z)=f(r z) / r$, belongs to class $\mathcal{H}$ for all $0<r \leq \rho$. Among the radius problems for various subclasses of analytic functions, one direction of study focuses on obtaining the radius for classes consisting of functions characterised by ratio of the function $f$ and another function $g$, where $g$ is a function belonging to some special subclass of $\mathcal{A}$ of all analytic functions on $\mathbb{D}$ normalized by $f(0)=0=f^{\prime}(0)-1$. MacGregor [7,8] obtained the radius of starlikeness for the class of functions $f \in \mathcal{A}$ satisfying either $\operatorname{Re}(f(z) / g(z))>0$ or $|f(z) / g(z)-1|<1$ for some $g \in \mathcal{K}$. Ali et al. 2] estimated several radii for classes of functions satisfying either (i) $\operatorname{Re}(f(z) / g(z))>0$, where $\operatorname{Re}(g(z) / z)>0$ or $\operatorname{Re}(g(z) / z)>1 / 2$; (ii) $|f(z) / g(z)-1|<1$, where $\operatorname{Re}(g(z) / z)>0$ or $g$ is convex; (iii) $\left|f^{\prime}(z) / g^{\prime}(z)-1\right|<1$, where $\operatorname{Re} g^{\prime}(z)>0$. The work is further investigated in [17. These classes are related to the Caratheodory class $\mathcal{P}$ consisting of all analytic functions $p$ with $p(0)=1$ and $\operatorname{Re} p(z)>0$ for all $z \in \mathbb{D}$.

Motivated by the aforesaid studies, we consider the following three classes $\mathcal{K}_{1}, \mathcal{K}_{2}$, and $\mathcal{K}_{3}$ :

$$
\begin{aligned}
& \mathcal{K}_{1}:=\left\{f \in \mathcal{A}: \frac{f(z)}{g(z)} \in \mathcal{P}, \text { for some } g \in \mathcal{A}, \operatorname{Re} \frac{1-z^{2}}{z} g(z)>0\right\}, \\
& \mathcal{K}_{2}:=\left\{f \in \mathcal{A}:\left|\frac{f(z)}{g(z)}-1\right|<1, \text { for some } g \in \mathcal{A}, \operatorname{Re} \frac{1-z^{2}}{z} g(z)>0\right\},
\end{aligned}
$$

and

$$
\mathcal{K}_{3}:=\left\{f \in \mathcal{A}: \operatorname{Re} \frac{1-z^{2}}{z} f(z)>0\right\},
$$

and estimate the radius for the functions in the classes to belong to various subclasses of starlike functions which we discuss below.

Let $f, F$ be analytic on $\mathbb{D}:=\{z \in \mathbb{C}:|z|<1\}$; the function $f$ is subordinate to $F$, written $f \prec F$, provided $f=F \circ w$ for some analytic self-mapping $w$ of the unit disk $\mathbb{D}$ that fixes the origin. Subordination is very useful in the study of subclasses of univalent functions. For instance, the concept of Hadamard product and subordination was used in 13 to introduce the class of all functions $f$ satisfying $z\left(k_{\alpha} * f\right)^{\prime} /\left(k_{\alpha} * f\right) \prec h$ where $k_{\alpha}(z)=z /(1-z)^{\alpha}, \alpha \in \mathbb{R}$, $f \in \mathcal{A}$ and $h$ is a convex function. Later in 1989, Shanmugam [19] studied the class $\mathcal{S}_{g}^{*}(h)$ of all functions $f \in \mathcal{A}$ satisfying $z(f * g)^{\prime} /(f * g) \prec h$ where $h$ is a convex function and $g$ is a fixed function in $\mathcal{A}$. By replacing $g$ with the functions $z /(1-z)$ and $z /(1-z)^{2}$, we get the subclasses $\mathcal{S}^{*}(h)$ and $\mathcal{K}(h)$ of Ma-Minda starlike and convex functions, respectively. In 1992, Ma and Minda 9] studied the distortion, growth, covering and coefficient estimates for

2010 Mathematics Subject Classification. 30C45, 30C80.

Key words and phrases. starlike functions, exponential function, lemniscate of Bernoulli, radius problems, coefficient estimate. 
these functions with the weaker assumption of starlikeness on $h$. These classes unifies several subclasses of starlike and convex functions. When $h$ is the mapping of $\mathbb{D}$ onto the right halfplane, $\mathcal{S}^{*}(h)$ and $\mathcal{K}(h)$ reduce to the class $\mathcal{S}^{*}$ of starlike and $\mathcal{K}$ of convex functions, respectively. For $h(z)=(1+A z) /(1+B z)$, with $-1 \leq B<A \leq 1$, the classes become $\mathcal{S}^{*}[A, B]$ of Janowski starlike functions and $\mathcal{K}[A, B]$ of Janowski convex functions. For $A=1-2 \alpha$ and $B=-1$ where $0 \leq \alpha<1$, these subclasses become $\mathcal{S}^{*}(\alpha)$ of the starlike functions of order $\alpha$ and $\mathcal{K}(\alpha)$ of convex functions of order $\alpha$, respectively introduced by Robertson [15. For $h(z)=\sqrt{1+z}$, the class $\mathcal{S}^{*}(h)$ becomes the class $\mathcal{S}_{L}^{*}$ of the lemniscate starlike functions introduced and studied by Sokól and Stankiewicz [20,21; analytically, $f \in \mathcal{S}_{L}^{*}$ if $\left|\left(z f^{\prime}(z) / f(z)\right)^{2}-1\right|<1$.

Mendiratta et al. [11,12] studied the classes $\mathcal{S}_{e}^{*}=\mathcal{S}^{*}\left(e^{z}\right)$ and $\mathcal{S}_{R L}^{*}=\mathcal{S}^{*}\left(h_{R L}\right)$, where

$$
h_{R L}:=\sqrt{2}-(\sqrt{2}-1) \sqrt{\frac{1-z}{1+2(\sqrt{2}-1) z}} .
$$

Indeed, a function $f$ belongs to $\mathcal{S}_{e}^{*}$ or to $\mathcal{S}_{R L}^{*}$ if $z f^{\prime}(z) / f(z)$ respectively belongs to $\{w \in \mathbb{C}$ : $|\log w|<1\}$ or $\left\{(w-\sqrt{2})^{2}-1<1\right\}$. Sharma et al. 23 defined and studied the class of functions defined by $\mathcal{S}_{c}^{*}=\mathcal{S}^{*}\left(h_{c}(z)\right)$, where $h_{c}(z)=1+(4 / 3) z+(2 / 3) z^{2}$; a function $f \in \mathcal{S}_{c}^{*}$ if $z f^{\prime}(z) / f(z) \in\left\{x+i y:\left(9 x^{2}+9 y^{2}-18 x+5\right)^{2}-16\left(9 x^{2}+9 y^{2}-6 x+1\right)=0\right\}$. Cho et al. 4] defined and studied the class $\mathcal{S}_{\sin }^{*}=\mathcal{S}^{*}(1+\sin z)$. Raina and Sokol [14] defined the class

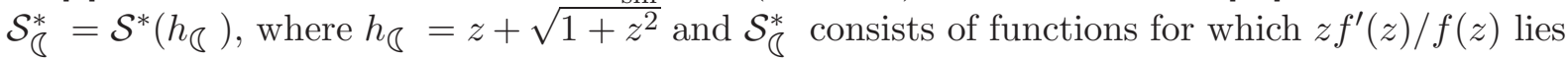

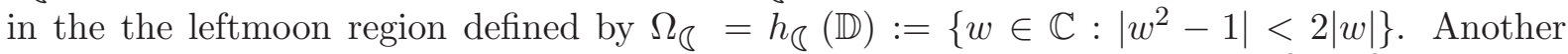
particular case is the class $\mathcal{S}_{R}^{*}=\mathcal{S}^{*}\left(h_{R}\right)$ studied in [6] where $h_{R}=1+\left(z k+z^{2}\right) /\left(k^{2}-k z\right)$, and $k=\sqrt{2}+1$. The subclass $\mathcal{S}_{P}$ of parabolic starlike functions (see the survey [16] or [1, 10, 22] ) consists of all normalized analytic functions $f$ with $z f^{\prime}(z) / f(z)$ lying in the parabolic region $(\operatorname{Im}(w))^{2}<2 \operatorname{Re}(w)-1$.

\section{Main Results}

The first theorem gives the various radii of starlikeness for the class $\mathcal{K}_{1}$ which consists of functions $f \in \mathcal{A}$ satisfying $\operatorname{Re}(f(z) / g(z))>0$ for some $g \in \mathcal{A}$ and $\operatorname{Re}\left(\left(1-z^{2}\right) g(z) / z\right)>0$. Note that the functions $f_{1}, g_{1}: \mathbb{D} \rightarrow \mathbb{C}$ defined by

$$
f_{1}(z)=\frac{z(1+i z)^{2}}{\left(1-z^{2}\right)(1-i z)^{2}} \quad \text { and } \quad g_{1}(z)=\frac{z(1+i z)}{\left(1-z^{2}\right)(1-i z)}
$$

satisfy

$$
\operatorname{Re} \frac{f_{1}(z)}{g_{1}(z)}=\operatorname{Re} \frac{1-z^{2}}{z} g_{1}(z)=\operatorname{Re} \frac{1+i z}{1-i z}>0 .
$$

This means the function $f_{1} \in \mathcal{K}_{1}$ and so $\mathcal{K}_{1} \neq \phi$. Further we will see that this function $f_{1}$ serves as an extremal function for many radii problems studied here.

Theorem 2.1. For the class $\mathcal{K}_{1}$, the following results hold:

(1) The $\mathcal{S}^{*}(\alpha)$-radius is the smallest positive real root of the equation $r^{4}(1+\alpha)-4 r^{3}-2 r^{2}-$ $4 r+(1-\alpha)=0, \quad 0 \leq \alpha<1$.

(2) The $\mathcal{S}_{L}^{*}$-radius is $R_{\mathcal{S}_{L}^{*}}=(\sqrt{5}-2) /(\sqrt{2}+1) \approx 0.0977826$.

(3) The $\mathcal{S}_{P}$-radius is the smallest positive real root of the equation $3 r^{4}-8 r^{3}-4 r^{2}-8 r+1=0$ i.e. $R_{\mathcal{S}_{P}} \approx 0.116675$.

(4) The $\mathcal{S}_{e}^{*}$-radius is the smallest positive real root of the equation $\left(2 r^{2}+4 r+4 r^{3}-1-r^{4}\right) e=$ $r^{4}-1$ i.e. $R_{\mathcal{S}_{e}^{*}} \approx 0.144684$.

(5) The $\mathcal{S}_{c}^{*}$-radius is the smallest positive real root of the equation $4 r^{4}-12 r^{3}-6 r^{2}-12 r+2=0$ i.e. $R_{\mathcal{S}_{c}^{*}} \approx 0.15182$.

(6) The $\mathcal{S}_{\mathbb{C}}^{*}$-radius is the smallest positive real root of the equation $4 r^{3}+2 r^{2}+4 r+\sqrt{2}\left(1-r^{4}\right)=$ 2 i.e. $R_{\mathcal{S}_{\overparen{\beta}}^{*}} \approx 0.134993$.

(7) The $\mathcal{S}_{\sin ^{*}}^{*}$ radius is $R_{\mathcal{S}_{\text {sin }}^{*}}=(-2+\sqrt{4+\sin 1(2+\sin 1)}) /(2+\sin 1) \approx 0.185835$.

(8) The $\mathcal{S}_{R L}^{*}$-radius is $R_{\mathcal{S}_{R L}^{*}} \approx 0.0687813$. 
(9) The $\mathcal{S}_{R}^{*}$-radius is the smallest positive real root of the equation $4 r^{3}+2 r^{2}+4 r-r^{4}-1=$ $2(1-\sqrt{2})\left(1-r^{4}\right)$ i.e. $R_{\mathcal{S}_{R}^{*}} \approx 0.0419413$.

All the radii obtained are sharp.

We would use the following lemmas in order to prove our results:

Lemma 2.2 ( [3, Lemma 2.2, p. 4]). For $0<a<\sqrt{2}$, let $r_{a}$ be given by

$$
r_{a}= \begin{cases}\left(\sqrt{1-a^{2}}-\left(1-a^{2}\right)\right)^{1 / 2}, & 0<a \leq 2 \sqrt{2} / 3 \\ \sqrt{2}-a, & 2 \sqrt{2} / 3 \leq a<\sqrt{2} .\end{cases}
$$

Then $\left\{w:|w-a|<r_{a}\right\} \subseteq\left\{w:\left|w^{2}-1\right|<1\right\}$.

Lemma 2.3 ( [19, Lemma 1, p. 321]). For a $>1 / 2$, let $r_{a}$ be given by

$$
r_{a}= \begin{cases}a-1 / 2, & 1 / 2<a \leq 3 / 2 \\ \sqrt{2 a-2}, & a \geq 3 / 2\end{cases}
$$

Then $\left\{w:|w-a|<r_{a}\right\} \subseteq\{w: \operatorname{Re} w>|w-1|\}=\Omega_{1 / 2}$. Here, $\Omega_{p}$ is a parabolic region which is symmetric with respect to the real axis and vertex at $(p, 0)$.

Lemma 2.4 ( [11, Lemma 2.2, p. 368]). For $e^{-1}<a<e$, let $r_{a}$ be given by

$$
r_{a}= \begin{cases}a-e^{-1}, & e^{-1}<a \leq\left(e+e^{-1}\right) / 2 ; \\ e-a, & \left(e+e^{-1}\right) / 2 \leq a<e .\end{cases}
$$

Then $\left\{w:|w-a|<r_{a}\right\} \subseteq\{w:|\log w|<1\}=\Omega_{e}$, which is the image of the unit disk $\mathbb{D}$ under the exponential function.

Lemma 2.5 ( [23, Lemma 2.5, p. 926]). For $1 / 3<a<3$, let $r_{a}$ be given by

$$
r_{a}= \begin{cases}(3 a-1) / 3, & 1 / 3<a \leq 5 / 3 \\ 3-a, & 5 / 3 \leq a \leq 3 .\end{cases}
$$

Then $\left\{w:|w-a|<r_{a}\right\} \subseteq \Omega_{c}$. Here $\Omega_{c}$ is the region bounded by the cardioid $\{x+i y$ : $\left.\left(9 x^{2}+9 y^{2}-18 x+5\right)^{2}-16\left(9 x^{2}+9 y^{2}-6 x+1\right)=0\right\}$.

Lemma 2.6 ( [4, Lemma 3.3, p. 7]). For $1-\sin 1<a<1+\sin 1$, let $r_{a}=\sin 1-|a-1|$. Then $\left\{w:|w-a|<r_{a}\right\} \subseteq \Omega_{\text {sin }}$. Here $\Omega_{\text {sin }}$ is the image of the unit disk $\mathbb{D}$ under the function $1+\sin z$.

Lemma 2.7 ( [5, Lemma 2.1, p. 3]). For $\sqrt{2}-1<a<\sqrt{2}+1$, let $r_{a}=1-|\sqrt{2}-a|$. Then $\left\{w:|w-a|<r_{a}\right\} \subseteq \Omega_{\S}=\left\{w:\left|w^{2}-1\right|<2|w|\right\}$.

Lemma 2.8 ( [6, Lemma 2.2, p. 202]). For $2(\sqrt{2}-1)<a<2$, let $r_{a}$ be given by

$$
r_{a}= \begin{cases}a-2(\sqrt{2}-1), & 2(\sqrt{2}-1)<a \leq \sqrt{2} \\ 2-a, & \sqrt{2} \leq a<2 .\end{cases}
$$

Then $\left\{w:|w-a|<r_{a}\right\} \subseteq \Omega_{R}$, where $\Omega_{R}$ is the image of the unit disk $\mathbb{D}$ under the function $1+\left(\left(z k+z^{2}\right) /\left(k^{2}-k z\right)\right), k=\sqrt{2}+1$.

Lemma 2.9 ( [12, Lemma 3.2, p. 10]). For $0<a<\sqrt{2}$, let $r_{a}$ be given by

$$
r_{a}= \begin{cases}a, & 0<a \leq \sqrt{2} / 3 ; \\ \left(\left(1-(\sqrt{2}-a)^{2}\right)^{1 / 2}-\left(1-(\sqrt{2}-a)^{2}\right)\right)^{1 / 2}, & \sqrt{2} / 3 \leq a<\sqrt{2} .\end{cases}
$$

Then $\left\{w:|w-a|<r_{a}\right\} \subseteq\left\{w: \operatorname{Re} w>0,\left|(w-\sqrt{2})^{2}-1\right|<1\right\}=\Omega_{R L}$.

Lemma 2.10 ( [18, Lemma 2, p. 240]). If $p(z)=1+b_{n} z^{n}+b_{n+1} z^{n+1}+\cdots$ is analytic and satisfies $\operatorname{Re} p(z)>\alpha, 0 \leq \alpha<1$, for $|z|<1$, then

$$
\left|\frac{z p^{\prime}(z)}{p(z)}\right| \leq \frac{2 n z^{n}(1-\alpha)}{\left(1-|z|^{n}\right)\left(1+(1-2 \alpha)|z|^{n}\right)} .
$$

With all these tools, we are ready to give the proof of our first result. 
Proof of Theorem 2.1. Let $f \in \mathcal{K}_{1}$ and the function $g: \mathbb{D} \rightarrow \mathbb{C}$ be chosen such that

$$
\operatorname{Re} \frac{f(z)}{g(z)}>0 \quad \text { and } \quad \operatorname{Re}\left(\frac{1-z^{2}}{z} g(z)\right)>0 \quad(z \in \mathbb{D}) .
$$

Let us define $p_{1}, p_{2}: \mathbb{D} \rightarrow \mathbb{C}$ as

$$
p_{1}(z)=\frac{1-z^{2}}{z} g(z) \quad \text { and } \quad p_{2}(z)=\frac{f(z)}{g(z)}
$$

Therefore, by equation (2.2), $p_{1}$ and $p_{2}$ are in $\mathcal{P}$. Equation (2.3) yields

$$
f(z)=\frac{z}{\left(1-z^{2}\right)} p_{1}(z) p_{2}(z) .
$$

Take logarithm at both sides and differentiate with respect to $z$ would give

$$
\frac{z f^{\prime}(z)}{f(z)}=\frac{1+z^{2}}{1-z^{2}}+\frac{z p_{1}^{\prime}(z)}{p_{1}(z)}+\frac{z p_{2}^{\prime}(z)}{p_{2}(z)} .
$$

It can be easily proved that the bilinear transform $w=\left(1+z^{2}\right) /\left(1-z^{2}\right)$ maps the disk $|z| \leq r$ onto the disk

$$
\left|\frac{1+z^{2}}{1-z^{2}}-\frac{1+r^{4}}{1-r^{4}}\right| \leq \frac{2 r^{2}}{1-r^{4}}
$$

Now, by Lemma 2.10, for $p \in \mathcal{P}(\alpha):=\{p \in \mathcal{P}: \operatorname{Re} p(z)>\alpha, z \in \mathbb{D}\}$, we have

$$
\left|\frac{z p^{\prime}(z)}{p(z)}\right| \leq \frac{2(1-\alpha) r}{(1-r)(1+(1-2 \alpha) r)} \quad(|z| \leq r) .
$$

By using equations (2.4), (2.5) and (2.6), we can conclude that a function $f \in \mathcal{K}_{1}$ maps the disk $|z| \leq r$ onto the disk

$$
\left|\frac{z f^{\prime}(z)}{f(z)}-\frac{1+r^{4}}{1-r^{4}}\right| \leq \frac{2 r\left(2 r^{2}+r+2\right)}{1-r^{4}} .
$$

In order to solve radius problems for $f \in \mathcal{K}_{1}$, we are interested in computing the value of $r$ for which the disk in (2.7) is contained in the corresponding regions. The classes we are considering here are all subclasses of starlike functions and therefore, we first determine the radius of starlikeness for $f \in \mathcal{K}_{1}$. From (2.7), we have

$$
\operatorname{Re} \frac{z f^{\prime}(z)}{f(z)} \geq \frac{r^{4}-4 r^{3}-2 r^{2}-4 r+1}{1-r^{4}} \geq 0
$$

Solving the above inequality for $r$, we get that the function $f \in \mathcal{K}_{1}$ is starlike in $|z| \leq 0.216845$. Hence, all the radii that we are going to estimate here, will be less than 0.216845 . For the function $f_{1}$ defined in (2.1), we have

$$
\begin{aligned}
\frac{z f_{1}^{\prime}(z)}{f_{1}(z)} & =\frac{1+4 i z+2 z^{2}-4 i z^{3}+z^{4}}{1-z^{4}} \\
& =\frac{1+4 i z\left(1-z^{2}\right)+2 z^{2}+z^{4}}{1-z^{4}}
\end{aligned}
$$

At $z:=r i=(0.216845) i$, we have $z f_{1}^{\prime}(z) / f_{1}(z) \approx 0$, thereby proving that the radius of starlikeness obtained for the class $\mathcal{K}_{1}$ is sharp.

(1) In order to compute $R_{\mathcal{S}^{*}(\alpha)}$, we estimate the value of $r \in(0,1)$ satisfying

$$
\operatorname{Re} \frac{z f^{\prime}(z)}{f(z)} \geq \frac{r^{4}-4 r^{3}-2 r^{2}-4 r+1}{1-r^{4}} \geq \alpha .
$$

Therefore, the number $r=R_{\mathcal{S}^{*}(\alpha)}$, is the smallest positive real root of the equation $r^{4}(1+\alpha)-4 r^{3}-2 r^{2}-4 r+(1-\alpha)=0$ in $(0,1)$. For the function $f_{1} \in \mathcal{K}_{1}$ given by 
(2.1), we have

$$
\frac{z f_{1}^{\prime}(z)}{f_{1}(z)}=\frac{1+4 i z+2 z^{2}-4 i z^{3}+z^{4}}{1-z^{4}}
$$

At $z:=r i=\mathcal{R}_{\mathcal{S}^{*}(\alpha)}$, (2.8) reduces to

$$
\frac{z f_{1}^{\prime}(z)}{f_{1}(z)}=\frac{1-4 r-2 r^{2}-4 r^{3}+r^{4}}{1-r^{4}}=\alpha
$$

thereby proving that the radius is sharp.

(2) We use lemma 2.2 to compute the lemniscate starlike radius for the function $f \in \mathcal{K}_{1}$. Let $a=\left(1+r^{4}\right) /\left(1-r^{4}\right)$. Then for $0 \leq r<1$, we have $a \geq 1$. So for $a<\sqrt{2}$, we get $r<\sqrt[4]{(\sqrt{2}-1) /(\sqrt{2}+2)} \approx 0.59018$. On the other hand, consider

$$
\frac{2 r\left(2 r^{2}+r+2\right)}{1-r^{4}} \leq \sqrt{2}-a=\sqrt{2}-\frac{1+r^{4}}{1-r^{4}}
$$

From this, let $r^{*}$ be the smallest positive real roof of the equation $(1+\sqrt{2}) r^{4}+4 r^{3}+$ $2 r^{2}+4 r+(1-\sqrt{2})=0$. Then the radius of lemniscate starlikeness for $f \in \mathcal{K}_{1}$ is

$$
R_{\mathcal{S}_{L}^{*}}=\min \left\{\left(\frac{\sqrt{2}-1}{\sqrt{2}+2}\right)^{1 / 4}, r^{*}\right\}=r^{*}=\frac{\sqrt{5}-2}{\sqrt{2}+1} .
$$

The radius obtained is sharp. Consider the functions $f, g: \mathbb{D} \rightarrow \mathbb{C}$ defined by

$$
f(z)=\frac{z(1-z)}{(1+z)^{3}} \quad \text { and } \quad g(z)=\frac{z}{(1+z)^{2}} .
$$

Then clearly $f \in \mathcal{K}_{1}$ as

$$
\operatorname{Re} \frac{f(z)}{g(z)}=\operatorname{Re} \frac{1-z^{2}}{z} g(z)=\operatorname{Re} \frac{1+z}{1-z}>0 .
$$

Now, for $z:=-r^{*}=-R_{\mathcal{S}_{L}^{*}}$, we have $\left(z^{2}-4 z+1\right) /\left(1-z^{2}\right)=\sqrt{2}$ and thus

$$
\left|\left(\frac{z f^{\prime}(z)}{f(z)}\right)^{2}-1\right|=\left|\left(\frac{r^{2}-4 r+1}{1-r^{2}}\right)^{2}-1\right|=1,
$$

thereby proving that the radius obtained is sharp by the function $f$ in (2.9) .

(3) We use Lemma 2.3 to compute the parabolic starlike radius for $f \in \mathcal{K}_{1}$. Again, let $a=\left(1+r^{4}\right) /\left(1-r^{4}\right)$, which is larger than or equal to 1 for $0 \leq r<1$. Note that

$$
a=\frac{1+r^{4}}{1-r^{4}}=\frac{3}{2} \quad \Leftrightarrow \quad r=\left(\frac{1}{5}\right)^{1 / 4} \approx 0.66874
$$

Since the radius we are looking for would be less than 0.216845 , we only consider the case $1 / 2<a \leq 3 / 2$ in Lemma 2.3. So when considering

$$
\frac{2 r\left(2 r^{2}+r+2\right)}{1-r^{4}} \leq \frac{1+r^{4}}{1-r^{4}}-\frac{1}{2}
$$

let $r^{*}$ be the smallest positive real root of the equation $3 r^{4}-8 r^{3}-4 r^{2}-8 r+1=0$. Then the radius of parabolic starlikeness for $f \in \mathcal{K}_{1}$ is

$$
R_{\mathcal{S}_{P}}=\min \left\{\left(\frac{1}{5}\right)^{1 / 4}, r^{*}\right\}=r^{*} \approx 0.116675 .
$$

We see that the sharpness follows for the function $f_{1} \in \mathcal{K}_{1}$ defined in (2.1). At $z=i r$, we have

$$
F(r)=\left.\frac{z f_{1}^{\prime}(z)}{f_{1}(z)}\right|_{z=i r}=\frac{1-4 r-2 r^{2}-4 r^{3}+r^{4}}{1-r^{4}}
$$


Then,

$$
|F(r)-1|=\left|\frac{2 r\left(r^{3}-2 r^{2}-r-2\right)}{1-r^{4}}\right| .
$$

For $z:=i r^{*}=i R_{\mathcal{S}_{P}}$, we have

$$
\begin{aligned}
\operatorname{Re} \frac{z f_{1}^{\prime}(z)}{f_{1}(z)} & =\frac{1+r^{4}-4 r^{3}-2 r^{2}-4 r}{1-r^{4}}(\approx 0.5) \\
& =\frac{2 r\left(2+r+2 r^{2}-r^{3}\right)}{1-r^{4}}=\left|\frac{z f_{1}^{\prime}(z)}{f_{1}(z)}-1\right| .
\end{aligned}
$$

Thus the radius obtained is sharp for the function $f_{1}$.

(4) By using Lemma 2.4 and the argument similar to the above, we get that the exponential starlike radius $R_{\mathcal{S}_{e}^{*}}$ for the class $\mathcal{K}_{1}$ is the smallest positive real root of the equation $\left(4 r^{3}+2 r^{2}+4 r-1-r^{4}\right) e=r^{4}-1$.

The radius is sharp for the function $f_{1}$ defined in (2.1). For $z:=i r=i R_{\mathcal{S}_{e}^{*}}$, we have

$$
\left|\log \frac{z f_{1}^{\prime}(z)}{f_{1}(z)}\right|=\left|\log \frac{1+r^{4}-4 r^{3}-2 r^{2}-4 r}{1-r^{4}}\right|=1 .
$$

(5) By using Lemma 2.5, and similar argument as before, the $\mathcal{S}_{c}^{*}$-radius for the class $\mathcal{K}_{1}$ is the smallest positive real root of the equation $2 r^{4}-6 r^{3}-3 r^{2}-6 r+1=0$.

The radius is sharp for the function $f_{1}$ defined in (2.1). Indeed, for the function $f_{1}$ defined in (2.1), we have at $z:=i r=i \mathcal{R}_{\mathcal{S}_{c}^{*}}$,

$$
\frac{z f_{1}^{\prime}(z)}{f_{1}(z)}=\frac{1+r^{4}-4 r^{3}-2 r^{2}-4 r}{1-r^{4}}=\frac{1}{3}=h_{c}(-1) \in \partial h_{c}(\mathbb{D}),
$$

where $h_{c}(z)=1+(4 / 3) z+(2 / 3) z^{2}$. This shows that the result is sharp.

(6) To determine the $\mathcal{S}_{\overparen{Q}}^{*}$-radius, $R_{\mathcal{S}_{\overparen{Q}}^{*}}$, we will use Lemma 2.7, After some computations following the idea above, it can be shown that $R_{\mathcal{S}_{\mathbb{S}}^{*}}$ is the smallest positive real root of the equation $4 r^{3}+2 r^{2}+4 r=2-\sqrt{2}\left(1-r^{4}\right)$.

The radius is sharp for the function $f_{1}$ defined in (2.1), since at $z:=i r=i R_{\mathcal{S}_{\mathbb{J}}^{*}}$, we have

$$
\begin{aligned}
\left|\left(\frac{z f_{1}^{\prime}(z)}{f_{1}(z)}\right)^{2}-1\right| & =\left|\left(\frac{1+r^{4}-4 r^{3}-2 r^{2}-4 r}{1-r^{4}}\right)^{2}-1\right|(\approx 0.134993) \\
& =2\left|\frac{1+r^{4}-4 r^{3}-2 r^{2}-4 r}{1-r^{4}}\right|=2\left|\frac{z f_{1}^{\prime}(z)}{f_{1}(z)}\right|
\end{aligned}
$$

(7) In order to find the $\mathcal{S}_{\sin ^{*}}$-radius for function $f \in \mathcal{K}_{1}$, we make use of Lemma 2.6. Similarly as above, with $a=\left(1+r^{4}\right) /\left(1-r^{4}\right)>1$, it can be shown by arguing similarly as above that the $\mathcal{S}_{\text {sin }}^{*}$-radius is the smallest positive real root of the equation $(2+\sin 1) r^{4}+4 r^{3}+$ $2 r^{2}+4 r-\sin 1=0$.

The radius is sharp for the function $f_{1}$ defined in (2.1).

(8) In order to compute the $\mathcal{S}_{R L^{-}}^{*}$ radius for the class $\mathcal{K}_{1}$, we use Lemma 2.9, As $\sqrt{2} / 3 \leq$ $a=\left(1+r^{4}\right) /\left(1-r^{4}\right)<\sqrt{2}$, a computation using Lemma 2.9 shows that the $\mathcal{S}_{R L^{-}}^{*}$ radius is the smallest positive real root of the equation

$$
4 r^{2}\left(2 r^{2}+r+2\right)^{2}=\left(1-r^{4}\right) \sqrt{(\sqrt{2}-1)+(\sqrt{2}-2) r^{4}}-2\left(\sqrt{2}-1+(\sqrt{2}-2) r^{4}\right) .
$$

The radius obtained is sharp for the function $f \in \mathcal{K}_{1}$ given by (2.9).

At $z:=-r=-R_{\mathcal{S}_{R L}^{*}}$, we have $\left(z^{2}-4 z+1\right) /\left(1-z^{2}\right)=\sqrt{2}$ and therefore,

$$
\left|\left(\frac{z f^{\prime}(z)}{f(z)}-\sqrt{2}\right)^{2}-1\right|=\left|\left(\frac{1-4 z+z^{2}}{1-z^{2}}-\sqrt{2}\right)^{2}-1\right|=1 \text {. }
$$


Hence the result.

(9) Since $2(\sqrt{2}-1)<a=\left(1+r^{4}\right) /\left(1-r^{4}\right) \leq \sqrt{2}$, by using Lemma 2.8, it can be shown that the $\mathcal{S}_{R^{-}}$radius is obtained by solving the equation

$$
(2 \sqrt{2}-1) r^{4}-4 r^{3}-2 r^{2}-4 r+(3-2 \sqrt{2})=0 .
$$

The radius is sharp for the function $f_{1}$ defined in (2.1).

Indeed, for the function $f_{1}$ defined in (2.1), we have at $z:=i r=i R_{\mathcal{S}_{R}^{*}}$ that

$$
\frac{z f_{1}^{\prime}(z)}{f_{1}(z)}=\frac{1+r^{4}-4 r^{3}-2 r^{2}-4 r}{1-r^{4}}=2 \sqrt{2}-2=h_{R}(-1) \in \partial h_{R}(\mathbb{D}) .
$$

Here, $h_{R}=1+\left(z k+z^{2}\right) /\left(k^{2}-k z\right)$, and $k=\sqrt{2}+1$.

Our next result gives various radii of starlikeness for the $\mathcal{K}_{2}$, which consists of functions $f \in \mathcal{A}$ satisfying $|(f(z) / g(z))-1|<1$ for some $g \in \mathcal{A}$ and $\operatorname{Re}\left(\left(1-z^{2}\right) g(z) / z\right)>0$. Consider the functions $f_{2}, g_{2}: \mathbb{D} \rightarrow \mathbb{C}$ defined by

$$
f_{2}(z)=\frac{z(1+i z)^{2}}{\left(1-z^{2}\right)(1-i z)} \quad \text { and } \quad g_{2}(z)=\frac{z(1+i z)}{\left(1-z^{2}\right)(1-i z)} .
$$

Clearly,

$$
\left|\frac{f_{2}(z)}{g_{2}(z)}-1\right|=|i z|=|z|<1 \quad \text { and } \quad \operatorname{Re} \frac{1-z^{2}}{z} g_{2}(z)=\operatorname{Re} \frac{1+i z}{1-i z}>0 .
$$

Therefore, the function $f_{2}$ is in $\mathcal{K}_{2}$ and this shows $\mathcal{K}_{2} \neq \phi$. Note that this function $f_{2}$ would serve as an extremal function for several radii-problems that we study here.

Theorem 2.11. For $f \in \mathcal{K}_{2}$, the following results hold:

(1) The sharp $\mathcal{S}^{*}(\alpha)$ radius is the smallest positive real root of the equation $\alpha r^{4}-3 r\left(r^{2}+\right.$ $r+1)+(1-\alpha)=0, \quad 0 \leq \alpha<1$.

(2) The $\mathcal{S}_{L}^{*}$ radius is $R_{\mathcal{S}_{\mathcal{L}}^{*}}=(\sqrt{2}-1) /(\sqrt{2}+2) \approx 0.12132$.

(3) The sharp $\mathcal{S}_{P}$ radius is the smallest positive real root of the equation $6 r^{3}+6 r^{2}+6 r-$ $1-r^{4}=0$ i.e., $R_{\mathcal{S}_{P}} \approx 0.1432698$.

(4) The sharp $\mathcal{S}_{e}^{*}$ radius is the smallest positive real root of the equation $\left(3 r^{3}+3 r^{2}+3 r-\right.$ 1) $e+1-r^{4}=0$ i.e., $R_{\mathcal{S}_{e}^{*}} \approx 0.174887$.

(5) The sharp $\mathcal{S}_{c}^{*}$ radius is the smallest positive real root of the equation $9 r^{3}+9 r^{2}+9 r-2-$ $r^{4}=0$ i.e., $R_{\mathcal{S}_{c}^{*}} \approx 0.182815$.

(6) The sharp $\mathcal{S}_{\mathbb{C}}^{*}$ radius is the smallest positive real root of the equation $r^{4}(1-\sqrt{2})+3 r^{3}+$ $3 r^{2}+3 r=2-\sqrt{2}$ i.e., $R_{\mathcal{S}_{\mathbb{Z}}^{*}} \approx 0.164039$.

(7) The sharp $\mathcal{S}_{\text {sin }}^{*}$ radius is $R_{\mathcal{S}_{\text {sin }}^{*}}=\sin 1 /(3+\sin 1) \approx 0.219049$.

(8) The sharp $\mathcal{S}_{R}^{*}$ radius is the smallest positive real root of the equation $2 r^{4}+3 r^{3}+3 r^{2}+$ $3 r-3+2 \sqrt{2}\left(1-r^{4}\right)=0$ i.e., $R_{\mathcal{S}_{R}^{*}} \approx 0.0541073$.

(9) The $\mathcal{S}_{R L}^{*}$ radius is $R_{\mathcal{S}_{R L}^{*}} \approx 0.0870259$.

Proof. Let $f \in \mathcal{K}_{2}$ and the function $g: \mathbb{D} \rightarrow \mathbb{C}$ be chosen such that

$$
\left|\frac{f(z)}{g(z)}-1\right|<1 \quad \text { and } \quad \operatorname{Re}\left(\frac{1-z^{2}}{z} g(z)\right)>0 \quad(z \in \mathbb{D}) .
$$

Note that $|f(z) / g(z)-1|<1$ holds if and only if $\operatorname{Re}(g(z) / f(z))>1 / 2$ Let define $p_{1}, p_{2}: \mathbb{D} \rightarrow \mathbb{C}$ as

$$
p_{1}(z)=\frac{1-z^{2}}{z} g(z) \quad \text { and } \quad p_{2}(z)=\frac{g(z)}{f(z)}
$$

Then, by equations (2.11) and (2.12), $p_{1} \in \mathcal{P}$ and $p_{2} \in \mathcal{P}(1 / 2)$. Equation (2.12) also yields

$$
f(z)=\frac{z}{1-z^{2}} \frac{p_{1}(z)}{p_{2}(z)} .
$$


Taking logarithm on both sides and differentiating with respect to $z$ gives

$$
\frac{z f^{\prime}(z)}{f(z)}=\frac{1+z^{2}}{1-z^{2}}+\frac{z p_{1}^{\prime}(z)}{p_{1}(z)}-\frac{z p_{2}^{\prime}(z)}{p_{2}(z)} .
$$

By using equations (2.5), (2.6) and (2.13), it can proven that the function $f$ maps the disk $|z| \leq r$ onto the disk

$$
\left|\frac{z f^{\prime}(z)}{f(z)}-\frac{1+r^{4}}{1-r^{4}}\right| \leq \frac{r\left(r^{3}+3 r^{2}+3 r+3\right)}{1-r^{4}}
$$

From (2.14), we can get

$$
\operatorname{Re} \frac{z f^{\prime}(z)}{f(z)} \geq \frac{1-3 r\left(r^{2}+r+1\right)}{1-r^{4}} \geq 0 .
$$

Upon solving for $r$, we can conclude that the function $f \in \mathcal{K}_{2}$ is starlike in $|z| \leq 0.253077 \cdots$. The classes we are considering here are all subclasses of starlike functions, hence, all the radii we estimate here, will be less than $0.253077 \cdots$. For the function $f_{2}$ defined in (2.10), we have

$$
\begin{aligned}
\frac{z f_{2}^{\prime}(z)}{f_{2}(z)} & =\frac{1+3 i z+3 z^{2}-3 i z^{3}}{1-z^{4}} \\
& =\frac{1+3 i z\left(1-z^{2}\right)+3 z^{2}}{1-z^{4}}
\end{aligned}
$$

At $z:=i r=i(0.253077)$, we have $z f_{2}^{\prime}(z) / f_{2}(z) \approx 0$, thereby proving that the radius of starlikeness obtained for the class $\mathcal{K}_{2}$ is sharp.

(1) In order to compute $R_{\mathcal{S}^{*}(\alpha)}$, we estimate the value of $r \in[0,1]$ satisfying

$$
\operatorname{Re} \frac{z f^{\prime}(z)}{f(z)} \geq \frac{1-3 r\left(r^{2}+r+1\right)}{\left(1-r^{4}\right)} \geq \alpha .
$$

Therefore, the number $R_{\mathcal{S}^{*}(\alpha)}$, is the root of the equation $\alpha r^{4}-3 r\left(r^{2}+r+1\right)+(1-\alpha)=0$ in $[0,1]$. For the function $f_{2} \in \mathcal{K}_{2}$ given by (2.10), we have

$$
\frac{z f_{2}^{\prime}(z)}{f_{2}(z)}=\frac{1+3 i z+3 z^{2}-3 i z^{3}}{1-z^{4}}
$$

At $z:=i r=R_{\mathcal{S}^{*}(\alpha)}$, 2.15) reduces to

$$
\frac{z f_{2}^{\prime}(z)}{f_{2}(z)}=\frac{1-3 r-3 r^{2}-3 r^{3}}{1-r^{4}}=\alpha,
$$

thereby proving that the radius is sharp.

(2) We would use Lemma 2.2 to compute the lemniscate starlike radius for $f \in \mathcal{K}_{2}$. So, let $a=\left(1+r^{4}\right) /\left(1-r^{4}\right)$. Then $1 \leq a<\infty$ for $r \in[0,1)$, and $a<\sqrt{2}$ when $r<$ $((\sqrt{2}-1) /(\sqrt{2}+1))^{1 / 4}$. From 2.14, we know that $f \in \mathcal{K}_{2}$ maps the disk $|z| \leq r$ onto the disk

$$
\left|\frac{z f^{\prime}(z)}{f(z)}-\frac{1+r^{4}}{1-r^{4}}\right| \leq \frac{r\left(r^{3}+3 r^{2}+3 r+3\right)}{1-r^{4}} .
$$

So, consider

$$
\frac{r\left(r^{3}+3 r^{2}+3 r+3\right)}{1-r^{4}} \leq \sqrt{2}-\frac{1+r^{4}}{1-r^{4}}
$$

and let $r^{*}$ be the smallest positive real root of the equation

$$
(\sqrt{2}+2) r^{4}-4 r^{3}-2 r^{2}-4 r+(3-2 \sqrt{2})=0 .
$$

Then by Lemma 2.2, the lemniscate starlike radius $R_{\mathcal{S}_{L}^{*}}$ for $f \in \mathcal{K}_{2}$ is given by

$$
R_{\mathcal{S}_{L}^{*}}=\min \left\{\left(\frac{\sqrt{2}-1}{\sqrt{2}+1}\right)^{1 / 4}, r^{*}\right\}=r^{*}=\frac{\sqrt{2}-1}{\sqrt{2}+2}=0.12132 \ldots
$$


This radius may not be sharp.

(3) We use Lemma 2.3 to compute the parabolic starlike radius for $f \in \mathcal{K}_{2}$. For $a=$ $\left(1+r^{4}\right) /\left(1-r^{4}\right)$, we have $a \leq 3 / 2$ if $r \leq(1 / 5)^{1 / 4} \approx 0.668740305$. by Lemma 2.3, consider

$$
\frac{r\left(r^{3}+3 r^{2}+3 r+3\right)}{1-r^{4}} \leq \frac{1+r^{4}}{1-r^{4}}-\frac{1}{2}
$$

and let $r^{*}$ be the smallest positive real root of the equation

$$
r^{4}-6 r^{3}-6 r^{2}-6 r+1=0 .
$$

Then the $\mathcal{S}_{P \text {-radius is }}$

$$
R_{\mathcal{S}_{P}}=\min \left\{\left(\frac{1}{5}\right)^{1 / 4}, r^{*}\right\}=r^{*} \approx 0.1432698 .
$$

We see that sharpness follows for the function $f_{2} \in \mathcal{K}_{2}$ defined in (2.10). As shown previously, at $z:=i r$, we have

$$
\frac{z f_{2}^{\prime}(z)}{f_{2}(z)}=\frac{1-3 r-3 r^{2}-3 r^{3}}{1-r^{4}}
$$

Thus,

$$
\left|\frac{z f_{2}^{\prime}(z)}{f_{2}(z)}-1\right|=\left|\frac{r\left(3+3 r+3 r^{2}-r^{3}\right)}{1-r^{4}}\right|
$$

For $z:=i r=i R_{\mathcal{S}_{P}}$, we have

$$
\begin{aligned}
\operatorname{Re} \frac{z f_{2}^{\prime}(z)}{f_{2}(z)} & =\frac{1-3 r-3 r^{2}-3 r^{3}}{1-r^{4}}(\approx 0.5) \\
& =\frac{r\left(3+3 r+3 r^{2}-r^{3}\right)}{1-r^{4}}=\left|\frac{z f_{2}^{\prime}(z)}{f_{2}(z)}-1\right| .
\end{aligned}
$$

Thus the radius obtained is sharp for the function $f_{2}$.

(4) For the $\mathcal{S}_{e}$-radius of $f \in \mathcal{K}_{2}$, we will use Lemma 2.4 since if $a=\left(1+r^{4}\right) /\left(1-r^{4}\right), 0 \leq r<1$, we have $a<e$ for $r<[(e-1) /(e+1)]^{1 / 4} \approx 0.82449$. Also, since $a \leq \frac{1}{2}\left(e+e^{-1}\right)$ for $r<[(e-1) /(e+1)]^{2} \approx 0.213552$, consider

$$
\frac{r\left(r^{3}+3 r^{2}+3 r+3\right)}{1-r^{4}} \leq \frac{1+r^{4}}{1-r^{4}}-\frac{1}{e}
$$

and let $r^{*}$ be the smallest positive real root of the equation

$$
r^{4}-e\left(3 r^{3}+3 r^{2}+3 r\right)+e-1=0 .
$$

Then the $\mathcal{S}_{e}$-radius is

$$
\begin{aligned}
& R_{\mathcal{S}_{e}}=\min \left\{\left(\frac{e-1}{e+1}\right)^{2}, r^{*}\right\}=r^{*} \approx 0.174887 . \\
& \left|\log \frac{z f_{2}^{\prime}(z)}{f_{2}(z)}\right|=\left|\log \frac{1-3 r-3 r^{2}-3 r^{3}}{1-r^{4}}\right|=1,
\end{aligned}
$$

hence proving that the exponential starlike radius obtained for the class $\mathcal{K}_{2}$ is sharp.

(5) By using Lemma 2.5. it can be proven similarly as above that the $\mathcal{S}_{c}^{*}$-radius $R_{\mathcal{S}_{c}^{*}}$ for the class $\mathcal{K}_{2}$ is the smallest positive real root of the equation $9 r^{3}+9 r^{2}+9 r-2-r^{4}=0$, which is $R_{\mathcal{S}_{c}^{*}} \approx 0.182815$.

The radius obtained is sharp for the function $f_{2}$ defined in (2.10) as for $z:=i r=i R_{\mathcal{S}_{c}^{*}}$,

$$
\frac{z f_{2}^{\prime}(z)}{f_{2}(z)}=\frac{1-3 r-3 r^{2}-3 r^{3}}{1-r^{4}}=\frac{1}{3}=h_{c}(-1) \in \partial h_{c}(\mathbb{D}) \text {. }
$$


(6) The $\mathcal{S}_{\mathbb{\complement}}^{*}$-radius $R_{\mathcal{S}_{\mathbb{\zeta}}^{*}}$ for the class $\mathcal{K}_{2}$ is the smallest positive real root of the equation $r^{4}(1-\sqrt{2})+3 r^{3}+3 r^{2}+3 r=2-\sqrt{2}$. This can be obtained by considering the inequality

$$
\frac{3 r^{3}+3 r^{2}+3 r-1}{1-r^{4}} \leq 1-\sqrt{2}
$$

and then using Lemma 2.7 ,

The radius is sharp for the function $f_{2}$ defined in (2.10), since at $z:=i r=i R_{\mathcal{S}_{\mathbb{J}}^{*}}$, we have

$$
\begin{aligned}
\left|\left(\frac{z f_{2}^{\prime}(z)}{f_{2}(z)}\right)^{2}-1\right| & =\left|\left(\frac{1-3 r-3 r^{2}-3 r^{3}}{1-r^{4}}\right)^{2}-1\right| \quad(\approx 0.82842) \\
& =2\left|\frac{1-3 r-3 r^{2}-3 r^{3}}{1-r^{4}}\right|=2\left|\frac{z f_{2}^{\prime}(z)}{f_{2}(z)}\right| .
\end{aligned}
$$

(7) In order to find the $\mathcal{S}_{\text {sin }}^{*}$-radius for the function $f \in \mathcal{K}_{2}$, we make use of Lemma 2.6, It is easy to see that $1-\sin 1<a=\left(1+r^{4}\right) /\left(1-r^{4}\right)<1+\sin 1$ for $r<[(\sin 1) /(2+\sin 1)]^{1 / 4}$. Since $a>1$, consider

$$
\frac{r\left(r^{3}+3 r^{2}+3 r+3\right)}{1-r^{4}} \leq \sin 1-\left(\frac{1+r^{4}}{1-r^{4}}-1\right) .
$$

Then the $\mathcal{S}_{\sin ^{*}}^{*}$-radius, $R_{\mathcal{S}_{\sin }^{*}}(\approx 0.219049)$, is the smallest positive real root of the equation

$$
(3+\sin 1) r^{4}+3 r\left(r^{2}+r+1\right)=\sin 1 .
$$

The radius obtained is sharp for the function $f_{2}$ defined in (2.10).

(8) We use Lemma 2.8 in order to compute the $\mathcal{S}_{R^{-}}^{*}$ radius for the class $\mathcal{K}_{2}$. Since $2(\sqrt{2}-1)<$ $a=\left(1+r^{4}\right) /\left(1-r^{4}\right) \leq \sqrt{2}$ for $r<[(\sqrt{2}-1) /(\sqrt{2}+1)]^{1 / 4} \approx 0.64359$, by Lemma 2.8 , we consider

$$
\frac{r\left(r^{3}+3 r^{2}+3 r+3\right)}{1-r^{4}} \leq \frac{1+r^{4}}{1-r^{4}}-2(\sqrt{2}-1) .
$$

Then the $\mathcal{S}_{R^{-}}^{*}$ radius for $\mathcal{K}_{2}$ can be computed to be $R_{\mathcal{S}_{R}^{*}} \approx 0.0870259$. The radius obtained is sharp for the function $f_{2}$ defined in (2.10). Indeed, for the function $f_{2}$ defined in (2.10), we have at $z:=i r=i R_{\mathcal{S}_{R}^{*}}$,

$$
\frac{z f_{2}^{\prime}(z)}{f_{2}(z)}=\frac{1-3 r-3 r^{2}-3 r^{3}}{1-r^{4}}=2 \sqrt{2}-2=h_{R}(-1) \in \partial h_{R}(\mathbb{D}) .
$$

This shows that the result is sharp.

(9) Finally, for the $\mathcal{S}_{R L^{-}}^{*}$ radius, $R_{\mathcal{S}_{R L}^{*}}$, for the class $\mathcal{K}_{2}$, by Lemma 2.9 the value of $R_{\mathcal{S}_{R L}^{*}} \approx$ 0.0541073 is obtained from solving the equation

$$
\begin{aligned}
\left(1-r^{4}\right)\left\{\left(1-r^{4}\right)^{2}-((\sqrt{2}-1)-\right. & \left.\left.(\sqrt{2}+1) r^{4}\right)^{2}\right\}^{1 / 2}=\left(r^{4}+3 r^{3}+3 r^{2}+3 r\right)^{2} \\
& +\left(1-r^{4}\right)^{2}-\left((\sqrt{2}-1)-(\sqrt{2}+1) r^{4}\right)^{2} .
\end{aligned}
$$

The last theorem aims at computing the various radii of starlikeness for the function $f \in \mathcal{K}_{3}$ that satisfies $\operatorname{Re}\left(\left(1-z^{2}\right) f(z) / z\right)>0$. Consider the function $f_{3}: \mathbb{D} \rightarrow \mathbb{C}$ defined by

$$
f_{3}(z)=\frac{z(1+i z)}{\left(1-z^{2}\right)(1-i z)}
$$

Clearly,

$$
\operatorname{Re} \frac{\left(1-z^{2}\right)}{z} f_{3}(z)=\operatorname{Re} \frac{1+i z}{1-i z}>0 .
$$

Therefore, the function $f_{3} \in \mathcal{K}_{3}$ and $\mathcal{K}_{3} \neq \phi$. This function $f_{3}$ would serve as an extremal function for various radius problems in the following theorem.

Theorem 2.12. For $f \in \mathcal{K}_{3}$, the following results hold: 
(1) The sharp $\mathcal{S}^{*}(\alpha)$ radius is the smallest positive real root of the equation $(1+\alpha) r^{4}-$ $2 r\left(r^{2}+r+1\right)+(1-\alpha)=0, \quad 0 \leq \alpha<1$.

(2) The sharp $\mathcal{S}_{L}^{*}$ radius is $R_{\mathcal{S}_{\mathcal{L}}}=(\sqrt{2}-1) /(\sqrt{2}+1) \approx 0.171573$.

(3) The sharp $\mathcal{S}_{P}$ radius is the smallest positive real root of the equation $4 r^{3}+4 r^{2}+4 r-$ $1-3 r^{4}=0$ i.e. $R_{\mathcal{S}_{P}} \approx 0.2021347$.

(4) The sharp $\mathcal{S}_{e}^{*}$ radius is the smallest positive real root of the equation $\left(2 r^{3}+2 r^{2}+2 r-\right.$ $\left.1-r^{4}\right) e+1-r^{4}=0$ i.e. $R_{\mathcal{S}_{e}^{*}} \approx 0.244259$.

(5) The sharp $\mathcal{S}_{c}^{*}$ radius is the smallest positive real root of the equation $3 r^{3}+3 r^{2}+3 r-1-$ $2 r^{4}=0$ i.e. $R_{\mathcal{S}_{c}^{*}} \approx 0.254726$.

(6) The sharp $\mathcal{S}_{\mathbb{Q}}^{*}$ radius is the smallest positive real root of the equation $2 r^{3}+2 r^{2}+2 r-$ $\sqrt{2} r^{4}=2-\sqrt{2}$ i.e. $R_{\mathcal{S}_{\mathbb{Q}}^{*}} \approx 0.229877$.

(7) The sharp $\mathcal{S}_{\text {sin }}^{*}$ radius is $R_{\mathcal{S}_{\text {sin }}^{*}}=\sin 1 /(2+\sin 1) \approx 0.296139$.

(8) The sharp $\mathcal{S}_{R}^{*}$ radius is the smallest positive real root of the equation $r^{4}+2 r^{3}+2 r^{2}+$ $2 r-3+2 \sqrt{2}\left(1-r^{4}\right)=0$ i.e. $R_{\mathcal{S}_{R}^{*}} \approx 0.0790749$.

(9) The $\mathcal{S}_{R L}^{*}$ radius is $R_{\mathcal{S}_{R L}^{*}} \approx 0.125145$.

Proof. Let the function $f \in \mathcal{K}_{3}$. Then

$$
\operatorname{Re}\left(\frac{1-z^{2}}{z} f(z)\right)>0 \quad(z \in \mathbb{D}) .
$$

Define $p: \mathbb{D} \rightarrow \mathbb{C}$ as

$$
p(z)=\frac{1-z^{2}}{z} f(z)
$$

Therefore, by equation (2.18), we have $p \in \mathcal{P}$ and

$$
f(z)=\frac{z}{\left(1-z^{2}\right)} p(z) .
$$

From this, take logarithm on both sides and then differentiate with respect to $z$ :

$$
\frac{z f^{\prime}(z)}{f(z)}=\frac{1+z^{2}}{1-z^{2}}+\frac{z p^{\prime}(z)}{p(z)} .
$$

By using equations (2.5), (2.6) and (2.20), we can prove that the function $f$ maps the disk $|z| \leq r$ onto the disk

$$
\left|\frac{z f^{\prime}(z)}{f(z)}-\frac{1+r^{4}}{1-r^{4}}\right| \leq \frac{2 r\left(r^{2}+r+1\right)}{1-r^{4}} .
$$

In order to solve radius problems, we are interested in computing the value of $r$ for which the disk in (2.21) is contained in the corresponding regions. Again the classes we are considering here are all subclasses of starlike functions and therefore, are defined by the quantity $z f^{\prime}(z) / f(z)$ lying in some region in the right half plane. In particular, for $f$ to be in $\mathcal{S}^{*}$, we need

$$
\operatorname{Re} \frac{z f^{\prime}(z)}{f(z)} \geq \frac{1+r^{4}-2 r\left(r^{2}+r+1\right)}{1-r^{4}} \geq 0
$$

Thus the function $f \in \mathcal{K}_{3}$ is starlike in $|z| \leq 0.346014$. With this, now all the radii we estimate here shall be less than 0.346014 .

For the function $f_{3}$ defined in (2.17), we have

$$
\begin{aligned}
\frac{z f_{3}^{\prime}(z)}{f_{3}(z)} & =\frac{1+2 i z+2 z^{2}-2 i z^{3}+z^{4}}{1-z^{4}} \\
& =\frac{1+2 i z\left(1-z^{2}\right)+2 z^{2}+z^{4}}{1-z^{4}} .
\end{aligned}
$$

At $z:=i r=i(0.346014)$, we have $z f_{3}^{\prime}(z) / f_{3}(z) \approx 0$, thereby proving that the radius of starlikeness obtained for the class $\mathcal{K}_{3}$ is sharp. 
(1) To determine the radius $R_{\mathcal{S}^{*}(\alpha)}$ of starlikeness of order $\alpha$, we estimate the value of $r \in[0,1]$ satisfying

$$
\operatorname{Re} \frac{z f^{\prime}(z)}{f(z)} \geq \frac{1+r^{4}-2 r\left(r^{2}+r+1\right)}{1-r^{4}} \geq \alpha .
$$

Hence, $R_{\mathcal{S}^{*}(\alpha)}$ is the root of the equation $(1+\alpha) r^{4}-2 r\left(r^{2}+r+1\right)+(1-\alpha)=0$ in $[0,1]$. From (2.22), if $z:=i r=i R_{\mathcal{S}^{*}(\alpha)}$, then (2.22) reduces to

$$
\frac{z f_{3}^{\prime}(z)}{f_{3}(z)}=\frac{1-2 r-2 r^{2}-2 r^{3}+r^{4}}{1-r^{4}}=\alpha
$$

which shows that $f_{3}$ is the extremal function.

(2) We can use Lemma 2.2 to compute the lemniscate starlike radius for $f \in \mathcal{K}_{3}$. For $a=\left(1+r^{4}\right) /\left(1-r^{4}\right)$, we have $a<\sqrt{2}$ when $r<((\sqrt{2}-1) /(\sqrt{2}+1))^{1 / 4}$. By (2.14) and Lemma 2.2, consider

$$
\frac{2 r\left(r^{2}+r+1\right)}{1-r^{4}} \leq \sqrt{2}-\frac{1+r^{4}}{1-r^{4}}
$$

and let $r^{*}$ be the smallest positive real root of the equation

$$
(\sqrt{2}+1) r^{4}+2 r^{3}+2 r^{2}+2 r+(1-\sqrt{2})=0 .
$$

Hence, the lemniscate starlike radius $R_{\mathcal{S}_{L}^{*}}$ for $f \in \mathcal{K}_{3}$ is given by

$$
R_{\mathcal{S}_{L}^{*}}=\min \left\{\left(\frac{\sqrt{2}-1}{\sqrt{2}+1}\right)^{1 / 4}, r^{*}\right\}=r^{*}=\frac{1-\sqrt{2}}{1+\sqrt{2}}=0.1715728753 \ldots
$$

For the sharpness, consider the function $\hat{f}_{3}: \mathbb{D} \rightarrow \mathbb{C}$ defined by

$$
\hat{f}_{3}(z)=\frac{z}{(1+z)^{2}} \text {. }
$$

Clearly,

$$
\operatorname{Re} \frac{1-z^{2}}{z} \hat{f}_{3}(z)=\operatorname{Re} \frac{1-z}{1+z}>0
$$

So $\hat{f}_{3} \in \mathcal{K}_{3}$. Also

$$
\left|\left(\frac{z \hat{f}_{3}^{\prime}(z)}{\hat{f}_{3}(z)}\right)^{2}-1\right|=\left|\left(\frac{1-z}{1+z}\right)^{2}-1\right| .
$$

Now, for $z:=r=R_{\mathcal{S}_{L}^{*}}$, we have $(1-z) /(1+z)=\sqrt{2}$ and

$$
\left|\left(\frac{z \hat{f}_{3}^{\prime}(z)}{\hat{f}_{3}(z)}\right)^{2}-1\right|=\left|(\sqrt{2})^{2}-1\right|=1,
$$

Therefore, the radius obtained is sharp for the function $\hat{f}_{3}$.

(3) For the parabolic starlike radius for $f \in \mathcal{K}_{3}$, we would use Lemma 2.3. For $r \leq(1 / 5)^{1 / 4}$, we have $a=\left(1+r^{4}\right) /\left(1-r^{4}\right) \leq 3 / 2$, and if we consider

$$
\frac{2 r\left(r^{2}+r+1\right)}{1-r^{4}} \leq \frac{1+r^{4}}{1-r^{4}}-\frac{1}{2},
$$

then the $\mathcal{S}_{P}$-radius is given by

$$
R_{\mathcal{S}_{P}}=\min \left\{\left(\frac{1}{5}\right)^{1 / 4}, r^{*}\right\}=r^{*} \approx 0.2021347,
$$

where $r^{*}$ is the smallest positive real root of the equation $3 r^{4}-4 r^{3}-4 r^{2}-4 r+1=0$. 
The sharpness of the result follows for the function $f_{3}$ defined in (2.17). As shown previously, at $z:=i r$, we have

$$
\frac{z f_{3}^{\prime}(z)}{f_{3}(z)}=\frac{1-2 r-2 r^{2}-2 r^{3}+r^{4}}{1-r^{4}} .
$$

Then for $z:=i r=R_{\mathcal{S}_{P}}$, we have

$$
\begin{aligned}
\operatorname{Re} \frac{z f_{3}^{\prime}(z)}{f_{3}(z)} & =\frac{1-2 r-2 r^{2}-2 r^{3}+r^{4}}{1-r^{4}} \quad(=0.5) \\
& =\frac{2 r\left(1+r+r^{2}-r^{3}\right)}{1-r^{4}}=\left|\frac{z f_{3}^{\prime}(z)}{f_{3}(z)}-1\right|,
\end{aligned}
$$

thus illustrates the radius obtained is sharp for the function $f_{3}$.

(4) By using Lemma 2.4 and considering

$$
\frac{2 r\left(r^{2}+r+1\right)}{\left(1-r^{4}\right)} \leq \frac{1+r^{4}}{1-r^{4}}-\frac{1}{e},
$$

it can be proven similarly as above that the exponential starlike radius $R_{\mathcal{S}_{e}^{*}}$ for the class $\mathcal{K}_{3}$ is the smallest positive real root of the equation $\left(2 r^{3}+2 r^{2}+2 r-1-r^{4}\right) e+1-r^{4}=0$. Again, for the function $f_{3}$ given in (2.17), at $z:=i r=i R_{\mathcal{S}_{e}^{*}} \approx i(0.244259)$, we have

$$
\left|\log \frac{z f_{3}^{\prime}(z)}{f_{3}(z)}\right|=\left|\log \frac{1-2 r-2 r^{2}-2 r^{3}+r^{4}}{1-r^{4}}\right|=1,
$$

thereby proving that the result obtained is sharp.

(5) For the $\mathcal{S}_{c}^{*}$-radius for the class $\mathcal{K}_{3}$, we use Lemma 2.5 by considering

$$
\frac{2 r\left(r^{2}+r+1\right)}{1-r^{4}} \leq \frac{1+r^{4}}{1-r^{4}}-\frac{1}{3} .
$$

Then it can be proven similarly as above that $R_{\mathcal{S}_{c}^{*}}$ is the smallest positive real root of the equation

$$
3 r^{3}+3 r^{2}+3 r-1-2 r^{4}=0 .
$$

The radius obtained is sharp for the function $f_{3}$ defined in (2.17).

Indeed, for the function $f_{3}$, we have at $z:=i r=i R_{\mathcal{S}_{c}^{*}} \approx i(0.254716)$ that

$$
\frac{z f_{3}^{\prime}(z)}{f_{3}(z)}=\frac{1-2 r-2 r^{2}-2 r^{3}+r^{4}}{1-r^{4}}=\frac{1}{3}=h_{c}(-1) \in \partial h_{c}(\mathbb{D}) .
$$

(6) By proving similarly as above, the $\mathcal{S}_{\mathbb{\zeta}}^{*}$-radius for the class $\mathcal{K}_{3}$ is the smallest positive real root of the equation

$$
2 r^{3}+2 r^{2}+2 r-\sqrt{2} r^{4}=2-\sqrt{2}
$$

In this case, we would use Lemma 2.7 and consider

$$
\frac{2 r\left(r^{2}+r+1\right)}{1-r^{4}} \leq 1-\sqrt{2}+\frac{1+r^{4}}{1-r^{4}} .
$$

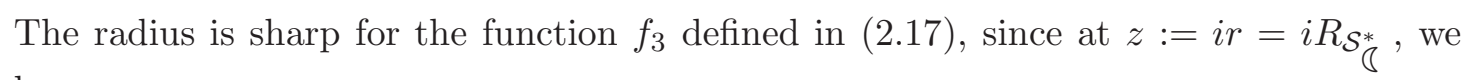
have

$$
\begin{aligned}
\left|\left(\frac{z f_{3}^{\prime}(z)}{f_{3}(z)}\right)^{2}-1\right| & =\left|\left(\frac{1-2 r-2 r^{2}-2 r^{3}+r^{4}}{1-r^{4}}\right)^{2}-1\right| \\
& =2\left|\frac{1-2 r-2 r^{2}-2 r^{3}+r^{4}}{1-r^{4}}\right|=2\left|\frac{z f_{3}^{\prime}(z)}{f_{3}(z)}\right| .
\end{aligned}
$$

(7) In order to find the $\mathcal{S}_{\text {sin }}^{*}$-radius for the function $f \in \mathcal{K}_{3}$, we make use of Lemma 2.6, where we would consider

$$
\frac{2 r\left(r^{2}+r+1\right)}{\left(1-r^{4}\right)} \leq \sin 1-\frac{2 r^{4}}{1-r^{4}} .
$$


The $\mathcal{S}_{\sin ^{*}}^{*}$-radius, $R_{\mathcal{S}_{\text {sin }}^{*}}$, is smallest positive real root of the equation

$$
2 r\left(r^{3}+r^{2}+r+1\right)=(\sin 1)\left(1-r^{4}\right) .
$$

The radius obtained is sharp for the function $f_{3}$ defined in (2.17).

(8) We use Lemma 2.8 to compute the $\mathcal{S}_{R}^{*}$-radius for the class $\mathcal{K}_{3}$. By considering

$$
\frac{2 r\left(r^{2}+r+1\right)}{1-r^{4}} \leq \frac{1+r^{4}}{1-r^{4}}-2(\sqrt{2}-1),
$$

we would obtain $R_{\mathcal{S}_{R}^{*}}$ to be given by the smallest positive real root of the equation

$$
(2 \sqrt{2}-1) r^{4}-2 r\left(r^{2}+r+1\right)+(3-2 \sqrt{2})=0 .
$$

The radius obtained is sharp for the function $f_{3}$ defined in (2.17).

Indeed, for the function $f_{3}$, we have at $z:=i r=i R_{\mathcal{S}_{c}^{*}} \approx 0.0790749$,

$$
\frac{z f_{3}^{\prime}(z)}{f_{3}(z)}=\frac{1-2 r-2 r^{2}-2 r^{3}+r^{4}}{1-r^{4}}=2 \sqrt{2}-2=h_{R}(-1) \in \partial h_{R}(\mathbb{D}) .
$$

(9) Lastly, the $\mathcal{S}_{R L^{-}}^{*}$ radius for the class $\mathcal{K}_{3}$ is obtained by using Lemma 2.9 and from the equation

$$
\begin{aligned}
\left(1-r^{4}\right)\left\{\left(1-r^{4}\right)^{2}-\left((\sqrt{2}-1)-(\sqrt{2}+1) r^{4}\right)^{2}\right\}^{1 / 2}= & \left(2 r^{3}+2 r^{2}+2 r\right)^{2}+\left(1-r^{4}\right)^{2} \\
& -\left((\sqrt{2}-1)-(\sqrt{2}+1) r^{4}\right)^{2}
\end{aligned}
$$

Acknowledgment. The second author gratefully acknowledges support from USM research university grants 1001.PMATHS.8011101.

\section{REFERENCES}

[1] R. M. Ali and V. Ravichandran, Uniformly convex and starlike functions, Ramanujan Mathematics Newsletter, 21 (2011), no. 1, 16-30.

[2] R. M. Ali, N. K. Jain and V. Ravichandran, On the radius constants for classes of analytic functions, Bull. Malays. Math. Sci. Soc. (2) 36 (2013), no. 1, 23-38.

[3] R. M. Ali, N. K. Jain and V. Ravichandran, Radii of starlikeness associated with the lemniscate of Bernoulli and the left-half plane, Appl. Math. Comput. 218 (2012), no. 11, 6557-6565.

[4] N. E. Cho et al., Radius problems for starlike functions associated with the sine function, Bull. Iranian Math. Soc. 45 (2019), no. 1, 213-232.

[5] S. Gandhi and V. Ravichandran, Starlike functions associated with a lune, Asian-Eur. J. Math. 10 (2017), no. 4, 1750064, 12 pp.

[6] S. Kumar and V. Ravichandran, A subclass of starlike functions associated with a rational function, Southeast Asian Bull. Math. 40 (2016), no. 2, 199-212.

[7] T. H. MacGregor, The radius of univalence of certain analytic functions, Proc. Amer. Math. Soc. 14 (1963), $514-520$

[8] T. H. MacGregor, The radius of univalence of certain analytic functions. II, Proc Amer. Math. Soc. 14 (1963), 521-524.

[9] W. C. Ma and D. Minda, A unified treatment of some special classes of univalent functions, in Proceedings of the Conference on Complex Analysis (Tianjin, 1992), 157-169, Conf. Proc. Lecture Notes Anal., I, Int. Press, Cambridge,MA.

[10] W. C. Ma and D. Minda, Uniformly convex functions, Ann. Polon. Math. 57 (1992), no. 2, $165-175$.

[11] R. Mendiratta, S. Nagpal and V. Ravichandran, On a subclass of strongly starlike functions associated with exponential function, Bull. Malays. Math. Sci. Soc. 38 (2015), no. 1, 365-386.

[12] R. Mendiratta, S. Nagpal and V. Ravichandran, A subclass of starlike functions associated with left-half of the lemniscate of Bernoulli, Internat. J. Math. 25 (2014), no. 9, 1450090, 17 pp.

[13] K. S. Padmanabhan and R. Parvatham, Some applications of differential subordination, Bull. Austral. Math. Soc. 32 (1985), no. 3, 321-330.

[14] R. K. Raina and J. Sokół, Some properties related to a certain class of starlike functions, C. R. Math. Acad. Sci. Paris 353 (2015), no. 11, 973-978.

[15] M. S. Robertson, Certain classes of starlike functions, Michigan Math. J. 32 (1985), no. 2, 135-140.

[16] F. Rønning, A survey on uniformly convex and uniformly starlike functions, Ann. Univ. Mariae CurieSkłodowska Sect. A 47 (1993), 123-134.

[17] A. Sebastian and V. Ravichandran, Radius of starlikeness of certain analytic functions, Math. Slovaca (accepted)

[18] G. M. Shah, On the univalence of some analytic functions, Pacific J. Math. 43 (1972), 239-250. 
[19] T. N. Shanmugam and V. Ravichandran, Certain properties of uniformly convex functions, in Computational methods and function theory 1994 (Penang), 319-324, Ser. Approx. Decompos., 5, World Sci. Publ., River Edge, NJ.

[20] J. Sokól and J. Stankiewicz, Radius of convexity of some subclasses of strongly starlike functions, Zeszyty Nauk. Politech. Rzeszowskiej Mat. No. 19 (1996), 101-105.

[21] J. Sokól, Radius problems in the class $\mathcal{S}_{L}^{*}$, Appl. Math. Comput. 214 (2009), no. 2, 569573.

[22] A. Gangadharan, V. Ravichandran and T. N. Shanmugam, Radii of convexity and strong starlikeness for some classes of analytic functions, J. Math. Anal. Appl. 211 (1997), no. 1, 301-313.

[23] K. Sharma, N. K. Jain and V. Ravichandran, Starlike functions associated with a cardioid, Afr. Mat. 27 (2016), no. 5-6, 923-939.

Department of Mathematics, SGTB Khalsa College, University of Delhi, Delhi-110 007, India E-mail address: kanika.khatter@yahoo.com

School of Mathematical Sciences, Universiti Sains Malaysia, 11800 USM, Penang, Malaysia E-mail address: sklee@usm.my

Department of Mathematics, Nit Tiruchirappalli, Tamil Nadu-620015, India

E-mail address: vravi68@gmail.com 